\section{Continuous accelerated radiotherapy effective in head and neck cancer}

Researchers from the Maria Sklodowska-Curie Memorial Institute, in Poland have previously reported the 3-year results of a randomized prospective trial that compared 7-days-a-week fractionation continuous accelerated irradiation (CAIR) with conventional 5-day fractionation in the treatment of moderately advanced head and neck cancer. Now, Skladowski et al. report the 5-year results of this trial.

From 374 consecutive patients with head and neck cancer, 100 patients with moderately advanced squamous cell carcinoma were randomized to conventional radiation treatment or CAIR. The overall treatment time for CAIR was 14 days shorter than for conventional treatment; all other dose-time parameters were identical. Actuarial 5-year local tumor control was significantly higher in the CAIR group than in the control group ( $75 \%$ vs $33 \% ; P=0.00002$ ). The increase in local tumor control in the CAIR group translated into significantly higher diseasefree and overall survival rates for these patients $(P=0.00001$ and $P=0.00005$, respectively). Mucositis was the chief acute toxicity in both treatment arms, although the incidence of this toxicity was significantly higher in the CAIR group $(P<0.00001)$. In addition, grade 4 toxicity developed in 5 of 23 patients in the CAIR group receiving 2.0 Gy fractions; however, no severe toxicity occurred when fraction size was reduced to $1.8 \mathrm{~Gy}$.

While highlighting the small study size, the authors conclude that CAIR with a fraction dose of $1.8 \mathrm{~Gy}$ can be an effective treatment regimen for certain patients with head and neck cancer.

Original article Skladowski K et al. (2006) Continuous accelerated 7-days-a-week radiotherapy for head-and-neck cancer: long-term results of phase III clinical trial. Int J Radiat Oncol Biol Phys 66: 706-713

\section{Paclitaxel is a suitable chemotherapy for patients with metastatic brain tumors}

In metastatic brain tumors (MBT), P-glycoprotein (Pgp) is abundantly expressed on the surface of cerebral capillaries and is responsible for the multidrug resistance phenomenon. In human cancer cell lines, tamoxifen, a nontoxic inhibitor of Pgp, was shown to inhibit the multidrug resistance phenotype. The objective of the study by Fine et al. was to assess differences in the deposition of paclitaxel between MBT and primary brain tumors (PBT) and to examine whether tamoxifen influenced the deposition of paclitaxel.

The trial included 27 patients with PBT or MBT who received intravenous paclitaxel $\left(175 \mathrm{mg} / \mathrm{m}^{2}\right.$ over $\left.3 \mathrm{~h}\right)$ either alone or with tamoxifen $\left(160 \mathrm{mg} / \mathrm{m}^{2}\right.$ orally on days $1-5$ preceding paclitaxel). The investigators showed that there was no significant difference between the two study groups regarding paclitaxel concentration in the tumor center, periphery or surrounding normal tissue. MBT showed greater average paclitaxel concentrations in the center (1.93-fold, $P=0.10)$ and periphery of the tumor (2.46-fold, $P=0.039)$ compared with PBT, which correlated with earlier studies showing decreased Pgp expression in MBT.

Although further studies using higher and longer dosages of tamoxifen should be performed, this work is the first to show increased deposition of paclitaxel in MBT versus PBT. The authors suggest that paclitaxel may be more suitable as a chemotherapeutic agent for MBT, dependent upon the tumor's origin and the clinical effectiveness of paclitaxel for the primary tumor's histopathology. Conceptually, this may lead to an expanded number of available natural product chemotherapy drugs for the treatment of MBT.

Original article Fine RL et al. (2006) Randomized study of paclitaxel and tamoxifen deposition into human brain tumors: implications for the treatment of metastatic brain tumors. Clin Cancer Res 12: 5770-5776

\section{Advanced breast cancer: aromatase inhibitors versus standard hormonal treatments}

Mauri and co-workers have performed a metaanalysis of randomized trials comparing aromatase inhibitors or inactivators (Als) with standard hormonal therapy in the treatment of advanced breast cancer, to determine whether Als offer a survival benefit. The trials covered the first-line, second-line (or subsequent-line) treatment of advanced breast cancer.

The analysis included 23 trials and 8,504 patients. The third-generation Als vorozole, 